\title{
Problems And Results on Colorings of Mixed HYPERGRAPHS
}

\author{
ZSOLT TUZA* and VITALY VOLOSHIN $^{\dagger}$
}

\begin{abstract}
We survey results and open problems on 'mixed hypergraphs' that are hypergraphs with two types of edges. In a proper vertex coloring the edges of the first type must not be monochromatic, while the edges of the second type must not be completely multicolored. Though the first condition just means 'classical' hypergraph coloring, its combination with the second one causes rather unusual behavior. For instance, hypergraphs occur that are uncolorable, or that admit colorings with certain numbers $k^{\prime}$ and $k^{\prime \prime}$ of colors but no colorings with exactly $k$ colors for any $k^{\prime}<k<k^{\prime \prime}$.
\end{abstract}

\section{INTRODUCTION}

In the classical theory of hypergraph coloring, colors have to be assigned to the vertices in such a way that no edge is colored completely with the same color. In this paper we consider a fruitful generalization of proper colorings, introduced in $[45,46]$. It turns out that under the more complex conditions involved, a more powerful model is obtained and, on the other hand, rather unusual phenomena arise. The goal of this paper is to provide an overview of results and open problems in this area.

Basic definitions. A mixed hypergraph is a triple $\mathcal{H}=(X, \mathcal{C}, \mathcal{D})$, where $X$ is the vertex set, and $\mathcal{C}$ and $\mathcal{D}$ are families of subsets of $X$, called the $\mathcal{C}$-edges

\footnotetext{
*Also affiliated with the Department of Computer Science, University of Pannonia, Veszprém, Hungary. Research supported in part by the Hungarian Scientific Research Fund, OTKA grant T-049613.

${ }^{\dagger}$ Partially supported by Troy University Research Grant.
} 
and $\mathcal{D}$-edges, respectively. A proper coloring of $\mathcal{H}$ is a mapping from $X$ into a set of $k$ colors so that each $\mathcal{C}$-edge has two vertices with a $\mathcal{C}$ ommon color and each $\mathcal{D}$-edge has two vertices with $\mathcal{D}$ istinct colors. A bi-edge is a vertex subset that is both a $\mathcal{C}$-edge and $\mathcal{D}$-edge.

A coloring may also be viewed as a partition of $X$, where the color classes (partition classes) are the sets of vertices assigned to the same color. Then the condition is that no class may contain a $\mathcal{D}$-edge, and each $\mathcal{C}$-edge has to meet some class in more than one vertex. A mixed hypergraph is $k$-colorable if it has a proper coloring with at most $k$ colors. A strict $k$-coloring is a proper $k$-coloring using all of the $k$ colors.

We obtain classical hypergraph coloring in the special case of $\mathcal{H}=$ $(X, \emptyset, \mathcal{D})$, which is denoted by $\mathcal{H}_{\mathcal{D}}$ and called a $\mathcal{D}$-hypergraph. A hypergraph $\mathcal{H}=(X, \mathcal{C}, \emptyset)$ will be denoted by $\mathcal{H}_{\mathcal{C}}$ and called a $\mathcal{C}$-hypergraph. Mixed hypergraphs with $\mathcal{C}=\mathcal{D}$ are called bi-hypergraphs.

The maximum number of colors in a strict coloring of $\mathcal{H}=(X, \mathcal{C}, \mathcal{D})$ is the upper chromatic number $\bar{\chi}(\mathcal{H})$; and the minimum number of colors is the lower chromatic number $\chi(\mathcal{H})$. Thus, general mixed hypergraphs represent structures where problems on both the minimum and maximum number of colors occur.

Some types of (mixed) hypergraphs. For a mixed hypergraph $\mathcal{H}$, a host graph is a graph $G$ on the same vertex set as $\mathcal{H}$, and such that every $\mathcal{C}$-edge and every $\mathcal{D}$-edge induces a connected subgraph in $G$. For a given $\mathcal{H}$, there can be many host graphs $G$. Depending on the type of $G$, particular terminology is used for $\mathcal{H}$ :

- If $G$ is a path, then $\mathcal{H}$ is called an interval hypergraph.

- If $G$ is a tree, then $\mathcal{H}$ is called a hypertree.

- If $G$ is a cycle, then $\mathcal{H}$ is called a circular hypergraph.

Let us mention further that $\mathcal{H}$ is called $r$-uniform if all of its $\mathcal{C}$ - and $\mathcal{D}$-edges have exactly $r$ vertices.

For general information and concepts not defined here, we refer to [3] on 'classical' hypergraphs and [47] on mixed hypergraphs; see also the regularly updated web site [48]. 


\section{Colorability Problem}

Although colorings are always possible in the classical or $\mathcal{C}$-hypergraph setting, the mixed hypergraphs in general may have no colorings at all. Hence, mixed hypergraphs can model not only extremal problems but also existence problems. In this way, the first problem that appears in mixed hypergraphs is to find out if they admit any coloring. This problem is called the colorability problem. A mixed hypergraph with no colorings at all is uncolorable [46]; otherwise it is called colorable.

The colorability problem represents a new type of problems in the theory of coloring. The structure of uncolorable mixed hypergraphs is unknown, and there is not much hope for a general description since the recognition problem of colorable mixed hypergraphs is already NP-complete for 3-uniform mixed hypergraphs (Tuza, Voloshin, Zhou [44]).

The first results concerning uncolorability were obtained by Tuza and Voloshin in [43]. There the existence of uncolorable mixed hypergraphs $\mathcal{H}=$ $(X, \mathcal{C}, \mathcal{D})$ having an arbitrarily large difference between $\bar{\chi}\left(\mathcal{H}_{\mathcal{C}}\right)$ and $\chi\left(\mathcal{H}_{\mathcal{D}}\right)$ was proven. More precisely, it was shown that for any $k=\bar{\chi}\left(\mathcal{H}_{\mathcal{C}}\right)-\chi\left(\mathcal{H}_{\mathcal{D}}\right)>$ 0 the minimum number of vertices of an inclusionwise minimal uncolorable mixed hypergraph is exactly $k+4$. (Minimality has to be assumed, since the trivial example of a 2-element bi-edge together with $k+1$ isolated vertices would yield a smaller bound.) This result also provides a clear evidence that, though $\bar{\chi}\left(\mathcal{H}_{\mathcal{C}}\right)<\chi\left(\mathcal{H}_{\mathcal{D}}\right)$ is a sufficient condition for uncolorability, it is very far from being necessary.

A measure of uncolorability, called the vertex uncolorability number, has been introduced [43]. It is the minimum number of vertices to be deleted in such a way that the mixed hypergraph obtained becomes colorable. A greedy algorithm - which is the first greedy mixed hypergraph coloring algorithm to determine an estimate on the vertex uncolorability number has also been developed.

It has been shown that the colorability problem can be expressed as an integer linear programming problem. There are two different formulations, the first one [43] involving the maximal independent sets of $\mathcal{H}_{\mathcal{C}}$ (hence, the description of its condition set may be exponentially large in terms of the input size), while the other one [34] reduces colorability to a polynomial-size integer linear program accepting $(0,1)$ solutions only. This latter model has been elaborated further to determine whether the input hypergraph admits a strict $k$-coloring $([34])$. 
A further connection to other fields is that the list colorability problem of graphs - where the existence of a proper coloring is a central issue represents a special case of the colorability problem on mixed hypergraphs without lists [31, 42, 43]. In fact, a large number of coloring problems on graphs can be modeled using mixed hypergraph coloring (Král' [27]).

Some particular classes of uncolorable mixed hypergraphs, too, were considered in [43], including e.g. the so-called complete $(l, m)$-uniform mixed hypergraphs; it means that all $l$-element subsets are viewed as $\mathcal{C}$-edges and all $m$-element subsets are $\mathcal{D}$-edges. The running parameter of the structure is the number $n$ of vertices. It was observed that for any fixed pair $(l, m)$, almost all complete $(l, m)$-uniform mixed hypergraphs are uncolorable, the largest colorable one having as few as $(l-1)(m-1)$ vertices. On the other hand, not fixing $l$ and $m$, generally almost all complete mixed hypergraphs are colorable, as $n$ gets large.

A subclass of uncolorable mixed hypergraphs can be derived from the class of $k$-chromatic graphs (graphs whose chromatic number is equal to $k$ ) [43], for any $k \geq 3$. In this construction the edges of a graph $G$ are taken as $\mathcal{D}$-edges of the hypergraph; and the $\mathcal{C}$-edges are the vertex sets of the $k$-vertex paths in $G$. Since $k$-critical graphs are hard to recognize, this example is a further indication that uncolorable mixed hypergraphs may have a rather complex structure in general.

Criteria of uncolorability for some special classes of mixed hypergraphs have been obtained, too. For example, a mixed hypertree is uncolorable if and only if it contains an 'evidently uncolorable' $\mathcal{D}$-edge, that is a $\mathcal{D}$ edge inside which each edge of the host tree is a 2-element $\mathcal{C}$-edge of the hypergraph [43]. In spite of the fact that the presence of an evidently uncolorable edge is 'evidently' sufficient for uncolorability, very little is known about classes of mixed hypergraphs where this condition is necessary, too.

\section{Open problems.}

1. Describe further structural properties and further subclasses of uncolorable mixed hypergraphs.

2. Search for the criteria of colorability for mixed hypergraphs derived from important classes, especially for 3-uniform bi-hypergraphs.

3. Investigate uncolorable mixed hypergraphs that are critical with respect to the deletion of vertices and/or edges. 
4. Determine the minimum value of $|\mathcal{C}|$, of $|\mathcal{D}|$, and of $|\mathcal{C}|+|\mathcal{D}|$, in inclusionwise minimal uncolorable $r$-uniform (bi-) hypergraphs on $n$ vertices, as a function of $n$ and $r$.

5. Develop algorithms for testing colorability and estimating the vertex uncolorability number. Results on the threshold concerning running time vs. the precision of approximation would be of great interest.

6. Characterize uncolorable mixed hypergraphs with vertex degree $k$, for $k \geq 2$ fixed.

\section{Uniquely Colorable Mixed Hypergraphs}

A mixed hypergraph is called uniquely colorable (UC, for short) if all of its proper colorings induce the same partition of the vertex set. The only uniquely colorable hypergraphs in classic coloring theory are the complete graphs (cliques). Thus, the uniquely colorable mixed hypergraphs are in this sense generalizations of cliques, and so they represent new 'absolutely rigid' combinatorial objects. But their structure is rather general. The first paper about uniquely colorable mixed hypergraphs is by Tuza, Voloshin and Zhou [44]. It is shown there, for example, that every mixed hypergraph having at least one coloring is an induced subhypergraph of some uniquely colorable mixed hypergraph. (Analogously to graph theory, the subhypergraph induced by a subset of vertices consists of all the $\mathcal{C}$-edges and $\mathcal{D}$-edges contained in the subset.)

Recursive formulas can be derived by introducing the concept of uniquely colorable separator (Voloshin, Zhou [50]). In this setting, $\mathcal{H}_{0}, \mathcal{H}_{1}, \mathcal{H}_{2}$ are assumed to be induced subhypergraphs of a mixed hypergraph $\mathcal{H}$, such that $\mathcal{H}_{1} \cup \mathcal{H}_{2}=\mathcal{H}, \mathcal{H}_{1} \cap \mathcal{H}_{2}=\mathcal{H}_{0}$, and $\mathcal{H}_{0}$ is uniquely colorable. Formulas have been presented that relate the upper and lower chromatic numbers of $\mathcal{H}_{0}, \mathcal{H}_{1}, \mathcal{H}_{2}$ with the upper and lower chromatic number of the original mixed hypergraph $\mathcal{H}$, and also the numbers of colorings of those three subhypergraphs with that of $\mathcal{H}$. The uniquely colorable separators open the way to build up new structures with interesting coloring properties, analogously to the way as chordal graphs are constructed from cliques.

It is easy to decide whether or not a graph is uniquely colorable, because in that case it is a clique. In general, however, it is algorithmically hard to determine if a mixed hypergraph is uniquely colorable. More precisely, given 
$\mathcal{H}$ together with one of its proper colorings, it is co-NP-complete to decide whether $\mathcal{H}$ is uniquely colorable (Tuza, Voloshin, Zhou [44]).

A weaker property has also been studied, where the mixed hypergraph has precisely one color partition with both $\bar{\chi}$ and $\chi$ colors, for $\bar{\chi}>\chi$ [44]. The class of these 'weakly uniquely colorable' mixed hypergraphs contains all uniquely colorable graphs in the usual graph-theoretic sense that requires a unique coloring with just $\chi$ colors (apart from the permutations of colors).

The following classes of uniquely colorable mixed hypergraphs have been characterized: those with $\chi=n-1$ and $\chi=n-2$ (Niculitsa, Voss [40]); UC mixed hypertrees (Niculitsa, Voloshin [39]); and UC circular mixed hypergraphs (Voloshin, Voss [49]). Moreover, pseudo-chordal mixed hypergraphs as a generalization of chordal graphs have been introduced and described by Voloshin and Zhou in [50]. Further, the possible size distributions of color classes in uniquely colorable $r$-uniform bi-hypergraphs have been characterized by Bacsó, Tuza, and Voloshin in [2].

A mixed hypergraph is UC-orderable [44] if there exists an ordering $x_{1}, x_{2}, \ldots, x_{n}$ of the vertex set such that every subhypergraph induced by an initial segment $\left\{x_{1}, \ldots, x_{i}\right\}$ of this ordering is uniquely colorable. It had been expected that this class of mixed hypergraphs could be efficiently recognized; but this has turned out to be false, as disproved by Bujtás and Tuza in [4]. As a matter of fact, testing UC-orderability is NP-complete. On the other hand, the possible color sequences of uniquely UC-orderable mixed hypergraphs have been characterized, and a linear-time algorithm for their recognition has been given [4]. Moreover, the UC-orderable mixed hypertrees have been completely characterized by Niculitsa and Voloshin in [39].

\section{Open problems.}

1. Search for conditions that are necessary or sufficient for a mixed hypergraph to be uniquely colorable.

2. Find the characterization of unique colorability for mixed hypergraphs derived from important classes, such as pseudo-chordal and planar mixed hypergraphs and those discussed in [3].

3. Search for conditions that are necessary or sufficient for a mixed hypergraph to be UC-orderable.

4. Determine the minimum value of $|\mathcal{C}|$, of $|\mathcal{D}|$, and of $|\mathcal{C}|+|\mathcal{D}|$, in uniquely colorable or UC-orderable or uniquely UC-orderable $r$-uniform (bi-) hypergraphs on $n$ vertices, as a function of $n$ and $r$. 
5. Develop algorithms for testing the unique colorability of mixed hypergraphs.

6. Characterize UC mixed hypergraphs with vertex degree $k$, for $k \geq 2$ fixed.

7. Characterize the structure of mixed hypergraphs whose UC-ordering is unique apart from transposing the first two vertices, and develop algorithms to recognize them.

\section{Chromatic Spectrum}

The set of values of $k$ for which $\mathcal{H}$ has a strict $k$-coloring is the feasible set. For each $k$, let $r_{k}$ denote the number of partitions of the vertex set into $k$ nonempty parts (color classes) such that the coloring constraint is satisfied on each $\mathcal{C}$ - and $\mathcal{D}$-edge. Such partitions are called feasible partitions. In fact, $r_{k}$ is strongly related to the number of strict $k$-colorings, with the only difference that in $r_{k}$ we do not count the permutations (re-labeling) of colors as distinct colorings. The vector $R(\mathcal{H})=\left(r_{1}, \ldots, r_{n}\right)$ is termed the chromatic spectrum $[45,46]$.

Given a colorable mixed hypergraph $\mathcal{H}$, it is natural and important to ask whether $\mathcal{H}$ has strict $k$-colorings for all $k$ such that $\chi(\mathcal{H}) \leq k \leq \bar{\chi}(\mathcal{H})$. Open since the introduction of mixed hypergraphs, this question has been solved in [23]. The answer is surprisingly negative: there may indeed be gaps in the chromatic spectrum.

A mixed hypergraph has a gap at $k$ if its feasible set contains elements larger and smaller than $k$ but omits $k$. A gap of size $g$ means $g$ consecutive gaps. If some gaps occur, the feasible set and the chromatic spectrum of $\mathcal{H}$ are said to be broken, and if there are no gaps then they are called continuous or gap-free.

A mixed hypergraph $\mathcal{H}_{s, t}$ has been constructed in [23] for all $2 \leq s \leq t-2$, that has feasible set $\{s, t\}$. Furthermore, it has been verified that $\mathcal{H}_{s, t}$ has the fewest vertices among all $s$-colorable mixed hypergraphs that have a gap at $t-1$; this minimum number of vertices is $2 t-s$. It follows, in particular, that $2 g+4$ is a tight lower bound on the number of vertices in mixed hypergraphs having a gap of size $g$. It has also been proven that a finite set of positive integers is a feasible set if and only if it is an initial interval $\{1, \ldots, t\}$ or does not contain the element 1 . This remains valid 
for $r$-uniform mixed- or bi-hypergraphs as well, except that no gaps smaller than $r$ can occur if all edges are required to have the same size $r$ (Bujtás, Tuza [6]). Constructions also show (Král [26]) that in the chromatic spectra of non-1-colorable mixed hypergraphs the sequence $\left(r_{2}, r_{3}, \ldots, r_{\bar{\chi}}\right)$ can take all combinations of nonnegative integers in its entries, except for the natural condition $r_{\bar{\chi}}>0$.

It is obvious that the feasible sets of all $r$-uniform $\mathcal{C}$-hypergraphs contain $\{1, \ldots, r-1\}$ as a subset. On $n$ vertices, the minimum number of $r$-element $\mathcal{C}$-edges to generate this smallest possible feasible set is $\lceil n(n-2) / 3\rceil$ in the particular case of $r=3$, but only some lower and upper estimates of the order $\Theta\left(n^{r-1}\right)$ are known if $r \geq 4$ (Diao et al. [14, 15]).

The chromatic spectrum of some special families of mixed hypergraphs has been investigated, too. In particular, it has been shown that no gaps can occur in mixed interval hypergraphs (Jiang et al. [23]), or more generally in hypertrees (Král' et al. [28]), and in mixed hypergraphs over a host graph in which any two cycles are vertex-disjoint (Král, Kratochvíl, Voss [30]). Gaps cannot arise either, if the mixed hypergraph has a uniquely colorable separator and the derived subhypergraphs do not have any gaps. This result implies that the so-called pseudo-chordal mixed hypergraphs [50] have gapfree chromatic spectra.

One of the important results states that mixed hypergraphs with maximum vertex degree 2 have no gaps (Král, Kratochvíl, Voss [29]). If we consider hypergraphs dual to these structures, then they are just multigraphs. In such multigraphs, we have vertices of two types: $\mathcal{C}$ - and $\mathcal{D}$-vertices. Now, in a proper coloring of the edges, we require that every $\mathcal{C}$-vertex be incident with at least two edges of the same color, and every $\mathcal{D}$-vertex be incident with at least two edges of different colors. Hence, in (multi)graphs, there is a natural way to introduce the concept of lower and upper chromatic indices, and the result states that proper colorings exist using every intermediate number of colors between minimum and maximum.

It is interesting to note that in the special case with just $\mathcal{C}$-vertices, an exact formula can be given: if such a multigraph has $n$ vertices, $m$ edges and $p$ pendant vertices, and $c$ denotes the maximum number of vertexdisjoint cycles in it, then the upper chromatic index is equal to $c+m-n+p$ (M. Gionfriddo, Milazzo, Voloshin [22]). This result may be viewed as a dual version of Vizing's celebrated theorem, with the remarkable aspect that in this 'mixed' case the graphs of 'class two' do not occur. 
Further notable facts in this direction are that some planar mixed hypergraphs have gaps (Kobler, Kündgen [24]), and mixed hypergraphs derived from some block designs may have gaps in their chromatic spectrum as well (L. Gionfriddo [20]); we return to these structures in a greater detail in Sections 6 and 7, respectively.

The discovery of gaps in the chromatic spectrum has far-reaching consequences in general coloring theory and its applications. As a matter of fact, mixed hypergraph colorings can model many combinatorial problems in a much more general context than graph colorings do. For example, they can model list colorings without lists, resource allocation, Ramsey-type problems, graph homomorphisms, etc., see [47, Chapter 12] and [27]. The presence or absence of gaps in the chromatic spectrum is vitally important in many of such applications.

\section{Open problems.}

1. Investigate the (non-) existence of gaps in the chromatic spectrum of further types of mixed hypergraphs.

2. Develop efficient algorithms to determine if the chromatic spectrum of mixed hypergraphs from some classes is gap-free.

3. Given a finite set $S$ of positive integers, determine or estimate the minimum and maximum numbers of $\left(\mathcal{C}_{-}, \mathcal{D}-\right.$, bi- $)$ edges in a mixed (bi-) hypergraph whose feasible set is $S$.

4. Is there a mixed hypergraph with gaps in the coefficients of its chromatic polynomial? ([47])

5. Investigate 'gap-critical' mixed hypergraphs; i.e., those with gaps in their chromatic spectrum, such that after deleting any edge some gaps disappear.

\section{Perfect Mixed Hypergraphs}

Every graph $G$ satisfies the trivial inequality $\chi(G) \geq \omega(G)$, where $\omega(G)$ is the size of the largest clique and $\chi(G)$ is the chromatic number in the usual sense. The perfect graphs are the graphs such that the equation $\chi\left(G^{\prime}\right)=\omega\left(G^{\prime}\right)$ holds for every induced subgraph $G^{\prime}$ of $G$. Many subclasses of perfect graphs have beautiful structural properties and admit fast algorithms for various optimization problems. 
A natural analogue of perfection for the upper chromatic number was introduced in [46]. In a mixed hypergraph, a set of vertices is $\mathcal{C}$-stable if it contains no $\mathcal{C}$-edges. The $\mathcal{C}$-stability number $\alpha_{\mathcal{C}}(\mathcal{H})$ is the maximum cardinality of a $\mathcal{C}$-stable set in $\mathcal{H}$. It is easy to see that the upper bound $\bar{\chi}(\mathcal{H}) \leq \alpha_{\mathcal{C}}(\mathcal{H})$ always is valid, because a set with more colors than $\alpha_{\mathcal{C}}(\mathcal{H})$ would assign distinct colors to all the vertices of some $\mathcal{C}$-edge. A mixed hypergraph $\mathcal{H}$ is called perfect $[46]$ if $\bar{\chi}\left(\mathcal{H}^{\prime}\right)=\alpha_{\mathcal{C}}\left(\mathcal{H}^{\prime}\right)$ holds for every induced subhypergraph $\mathcal{H}^{\prime}$. Notice that the perfection of graphs is related to the lower chromatic number, while the perfection of hypergraphs involves the upper chromatic number. In this setting, every $\mathcal{D}$-hypergraph (that is, hypergraph in the classical sense, without $\mathcal{C}$-edges) is perfect, because in each of its subhypergraphs - the upper chromatic number is equal to the number of vertices.

Several classes of perfect and minimal imperfect mixed hypergraphs have been found. A cycloid [46] is an $r$-uniform $\mathcal{C}$-hypergraph on $n$ vertices, denoted by $\mathcal{C}_{n}^{r}$, which has $n \mathcal{C}$-edges and admits a simple cycle on $n$ vertices as a host graph. A polystar is a mixed hypergraph with at least two $\mathcal{C}$-edges, in which the set $Y$ of vertices common to all $\mathcal{C}$-edges (center) is nonempty, and every vertex pair in $Y$ forms a $\mathcal{D}$-edge. When the center consists of just one vertex, the polystar is also called monostar. Hence, every polystar in a $\mathcal{C}$-hypergraph is a monostar. A bistar (called co-bistar in [46]) is a mixed hypergraph in which there exists a pair of distinct vertices common to all $\mathcal{C}$-edges but not forming a $\mathcal{D}$-edge.

Bistars are perfect, while polystars are not [46]. Also, cycloids of the form $\mathcal{C}_{2 r-1}^{r}$ are not perfect [46]. Indeed, when $n=2 r-1$, we have $\alpha_{\mathcal{C}}\left(\mathcal{C}_{n}^{r}\right)=2 r-3$ and $\bar{\chi}\left(\mathcal{C}_{n}^{r}\right)=2 r-4$. These cycloids are analogous to the well-known minimal imperfect graphs. Polystars and cycloids of the form $\mathcal{C}_{2 r-1}^{r}, r \geq 3$, are minimal imperfect mixed hypergraphs in the sense that every proper induced subhypergraph of such a cycloid is perfect, and every subhypergraph of a polystar that is not a polystar is perfect. (A cycloid on fewer than $2 r-1$ vertices is perfect, whereas on more than $2 r-1$ vertices it contains a monostar, hence it is imperfect but not minimally imperfect.)

It was conjectured in [46] that an $r$-uniform $\mathcal{C}$-hypergraph is perfect if and only if it has no induced monostar or cycloid of the form $\mathcal{C}_{2 r-1}^{r}, r \geq 3$. These two natural imperfect families served as an analogue of Berge's Strong Perfect Graph Conjecture, which stated that a graph $G$ is perfect if and only if no odd cycle of length at least 5 occurs as an induced subgraph of $G$ or its complement $\bar{G}$ (proved recently by Chudnovsky et al. [13]). 
There are some classes of $\mathcal{C}$-hypergraphs for which the conjecture is true. For example, Bulgaru and Voloshin [12] proved that a mixed interval hypergraph is perfect if and only if it has no induced polystars. In [47] it was proved that if a mixed hypertree does not contain polystars as partial subhypergraphs, then it is perfect. It is clear that the situation is more complex than in case of graphs. (We are not formulating any guess about the complexity of a possible proof...)

Král [25] has disproved the mixed hypergraph perfection conjecture for each $r \geq 3$, by constructing a new family of minimal imperfect $\mathcal{C}$-hypergraphs (one $\mathcal{C}$-hypergraph for each $r$, on $2 r$ vertices) different from monostars and cycloids. Recently, Bujtás and Tuza [11] have found a larger family of counterexamples for $r \geq 4$, an increasing number of minimally imperfect $r$-uniform $\mathcal{C}$-hypergraphs as $r$ gets large.

Regarding the 3-uniform case, up to isomorphism the following six examples of minimal imperfect $\mathcal{C}$-hypergraphs are known:

- $V_{1}=(\{1,2,3,4\},\{\{1,2,3\},\{1,3,4\},\{1,2,4\}\})$ - monostar,

- $V_{2}=(\{1,2,3,4,5\},\{\{1,2,3\},\{1,4,5\}\})$ - monostar,

- $V_{3}=(\{1,2,3,4,5\},\{\{1,2,3\},\{1,3,4\},\{1,4,5\}\})$ - monostar,

- $V_{4}=(\{1,2,3,4,5\},\{\{1,2,3\},\{1,3,4\},\{1,4,5\},\{1,2,5\}\})-$ monostar,

- $V_{5}=(\{1,2,3,4,5\},\{\{1,2,3\},\{2,3,4\},\{3,4,5\},\{4,5,1\},\{5,1,2\}\})-$ cycloid $\mathcal{C}_{5}^{3}$,

- $K_{1}=(\{1,2,3,4,5,6\},\{\{1,2,4\},\{2,3,5\},\{3,4,6\},\{4,5,1\},\{5,6,2\}$, $\{6,1,3\},\{1,3,5\},\{2,4,6\}\})$ - Král's construction.

There may be other minimal imperfect 3-uniform mixed hypergraphs, as it is the case for $r \geq 4$. On the other hand, polystars generally are not uniform and they already indicate that the family of non-uniform minimal imperfect mixed hypergraphs may be complex. All these results and investigations will lead sooner or later to a more general conjecture about perfect mixed hypergraphs.

Algorithmic complexity aspects of perfection. It is well-known that perfection on graphs has led to efficient polynomial- and linear-time algorithms for solving several problems (not only coloring, but also maximumweight clique, minimum vertex cover, etc.) that are NP-complete in general. 
One can already see that a similar situation occurs with the perfection of hypergraphs.

All classes of perfect mixed hypergraphs known so far can be uppercolored efficiently. These include bistars, mixed interval hypergraphs (Bulgaru, Voloshin [12]) and quasi-interval $\mathcal{C}$-hypergraphs (Prisakaru [41]). These were the simplest cases of perfect mixed hypergraphs. As we have already mentioned, if a mixed hypertree does not contain monostars, then it is perfect. In this case there is an efficient polynomial algorithm (and it is even possible to develop a linear-time algorithm, too) for finding the upper chromatic number and a respective coloring. When monostars are allowed in $\mathcal{C}$-hypertrees, the problem is already NP-complete; it is NP-complete even for monostars themselves [18]. One may expect that perfection will lead to efficient polynomial-time algorithms for finding $\bar{\chi}$ and properly coloring the given hypergraph. In addition, perfection may serve as a hint for the search of efficient polynomial algorithms for other hard combinatorial problems on discrete structures.

\section{Open problems.}

1. Search for new classes of perfect and minimal imperfect mixed hypergraphs.

2. Describe classes of uniform $\mathcal{C}$-hypergraphs in which the exclusion of monostars and cycloids of certain lengths implies perfectness.

3. Develop efficient algorithms to compute the upper chromatic number, and to find maximum colorings, for various classes of perfect mixed hypergraphs.

4. Prove or disprove: A 3-uniform $\mathcal{C}$-hypergraph is perfect if and only if it does not contain any of the families $V_{1}-V_{5}$ and $K_{1}$ above as an induced subhypergraph.

\section{Planar Mixed Hypergraphs}

Let $\mathcal{H}=(X, \mathcal{E})$ be a hypergraph. The bipartite representation of $\mathcal{H}$, denoted by $B(\mathcal{H})$, is the bipartite graph with vertex set $X \cup \mathcal{E}$, where $x \in X$ is adjacent to $E \in \mathcal{E}$ in $B(\mathcal{H})$ if and only if $x \in E$ in $\mathcal{H}$. The following definition is due to Zykov [51]: a hypergraph $\mathcal{H}$ is called planar if $B(\mathcal{H})$ is a planar graph. 
In this way, planar graphs are the special cases of planar hypergraphs, in which all edges have size 2. As one can see, a planar hypergraph admits an embedding in the plane in such a way that each vertex corresponds to a point in the plane, and each edge corresponds to a closed region homeomorphic to a disk such that its boundary contains the points corresponding to its vertices, and it does not contain any points corresponding to the other vertices. Furthermore, two such regions intersect exactly in the points that correspond to the vertices in the intersection of the corresponding edges. In this way, the faces of the embedding of the planar hypergraph are formed by those connected regions of the plane which do not correspond to the edges.

Using properties of the bipartite representation $B(\mathcal{H})$, one can derive many properties of a planar embedding of the hypergraph $\mathcal{H}$. For example, denoting the degree of vertex $x \in X$ in $\mathcal{H}$ by $d_{\mathcal{H}}(x)$, we obtain the following generalization [32] of Euler's formula: for any planar embedding of $\mathcal{H}=$ $(X, \mathcal{E})$ with $f$ faces,

$$
|X|+|\mathcal{E}|-\sum_{E \in \mathcal{E}}|E|+f=|X|+|\mathcal{E}|-\sum_{x \in X} d_{\mathcal{H}}(x)+f=2 .
$$

In particular, the number of faces is independent of the embedding.

An embedding of a planar hypergraph is called maximal if every face (including the unbounded face, too) contains precisely two vertices; or, equivalently, if in the corresponding embedding of $B(\mathcal{H})$ every face is a cycle of length 4. A planar hypergraph is maximal if it has a maximal embedding in the plane. This maximality is relative in the sense that in every such face one can always insert a new edge of size 2 . However, if a planar hypergraph $\mathcal{H}$ is not maximal, then there is at least one face of size at least 3 , and therefore one can insert a new edge of size at least 3 inside that face.

If we draw the faces of a maximal planar hypergraph as curves connecting respective vertices, then we obtain a plane graph whose faces correspond to the edges of the initial hypergraph. In this way, we may look at a plane graph as a planar embedding of a maximal hypergraph such that the faces of the graph correspond to the edges of the hypergraph.

Let $\mathcal{H}=(X, \mathcal{C}, \mathcal{D})$ be a mixed hypergraph. Denote the underlying edge set of $\mathcal{H}$ by $\mathcal{E}=\mathcal{C} \cup \mathcal{D}$; if a $\mathcal{C}$-edge and a $\mathcal{D}$-edge consist of the same set of vertices (i.e., it is a bi-edge), then this set appears only once in $\mathcal{E}$. We say that $\mathcal{H}^{\prime}=(X, \mathcal{E})$ is the underlying hypergraph of $\mathcal{H}$.

A mixed hypergraph $\mathcal{H}=(X, \mathcal{C}, \mathcal{D})$ is planar if and only if its underlying hypergraph $\mathcal{H}^{\prime}$ is planar. This can be verified by embedding $\mathcal{H}^{\prime}$ in the plane 
and labeling each hyperedge with $B, C$, or $D$ appropriately, according to whether it is a bi-edge, $\mathcal{C}$-edge, or $\mathcal{D}$-edge. Note that $\mathcal{C}$-edges of size 2 can be contracted, and bi-edges of size 2 lead to uncolorability, so that in general it suffices to restrict our attention to mixed hypergraphs containing neither.

The question of coloring properties of general planar mixed hypergraphs was first raised in [46] (Problem 8, p. 43). This class already contains uncolorable members. The smallest non-trivial (reduced) uncolorable planar mixed hypergraph $\mathcal{H}=(X, \mathcal{C}, \mathcal{D})$ has three vertices and four edges: $X=$ $\{1,2,3\}, \mathcal{C}=\{\{1,2,3\}\}, \mathcal{D}=\{\{1,2\},\{1,3\},\{2,3\}\}$. One can easily embed it in the plane with 4 faces ( 3 of them containing 2 vertices each, and 1 containing 3 vertices). It is not difficult to extend this example to an infinite family of minimal uncolorable planar mixed hypergraphs.

The structure of uncolorable planar mixed hypergraphs is unknown. In general, allowing $\mathcal{D}$-edges of size 2 implies that the four-color problem is a special case of the theory of planar mixed hypergraphs. Therefore, it is reasonable to distinguish planar mixed hypergraphs without edges of size 2 from those containing edges of size 2 .

The first interesting case is where $\mathcal{H}=(X, \mathcal{C}, \mathcal{D})$ is a 3-uniform maximal planar bi-hypergraph. Since maximality means that every face is of size 2 , we can associate a graph $G(\mathcal{H})$ with $\mathcal{H}$, on the same vertex set: replace each face in $\mathcal{H}$ by an edge in $G$, so that every edge of $\mathcal{H}$ becomes a face of $G$. Since $\mathcal{H}$ is maximal 3-uniform, $G$ must be a triangulation in the usual sense. We call both $\mathcal{H}$ and $G$ bi-triangulations because every edge of $\mathcal{H}$ is a bi-edge.

Colorings of bi-triangulations have been investigated (Kündgen, Mendelsohn, Voloshin [32]). It has been proved that they are always colorable, the chromatic spectrum is gap-free, and, moreover, their chromatic polynomial has a very special form.

An important discovery in the coloring of planar mixed hypergraphs is that their chromatic spectrum may have gaps. Kobler and Kündgen's smallest example [24] has 6 vertices and its feasible set is $\{2,4\}$. Moreover, it is proved in [24] that a nonempty set $S$ of positive integers is the feasible set of some planar mixed hypergraph if and only if $S$ is an interval $\{s, s+1$, $\ldots, t\}$ with $1 \leq s \leq 4$ or is of the form $\{2,4,5, \ldots, t\}$. In other words, planar mixed hypergraphs may have gaps, but the gap can only occur at 3 .

\section{Open problems.}

1. Characterize the chromatic spectra of planar mixed hypergraphs. 
2. Search for the criteria of uncolorability and unique colorability for various subclasses of planar mixed hypergraphs (e.g., $r$-uniform, containing a fixed number of edges of size 2, pseudo-chordal, etc.)

3. Characterize perfect planar mixed hypergraphs.

\section{Coloring Block Designs}

For integers $v \geq k>t \geq 2$, a Steiner system $S(t, k, v)$ (of index 1) is a family $\mathcal{B}$ of $k$-element subsets - called blocks - over a $v$-element vertex set $X$, such that every $t$-subset of $X$ is contained in precisely one block. For the particular cases of $(t, k)=(2,3)$ and $(t, k)=(3,4)$, the notation $S T S(v)$ and $S Q S(v)$ are commonly used (Steiner triple systems and Steiner quadruple systems of order $v$, respectively).

When looking at Steiner systems as mixed hypergraphs, in principle one might decide on each block independently whether it is a $\mathcal{C}$-edge, or a $\mathcal{D}$-edge, or a bi-edge. Homogeneous conditions, however, are of primary interest. To distinguish between the three basic types, we use different notation.

- When all the blocks are regarded as $\mathcal{D}$-edges, we have a $\mathcal{D}$-hypergraph of the type $\mathcal{H}=(X, \emptyset, \mathcal{B})$. In this case, we keep the classic notation $S(t, k, v)$ or $S T S(v)$ or $S Q S(v)$.

- When all the blocks are regarded as $\mathcal{C}$-edges, we deal with a $\mathcal{C}$-hypergraph of the type $\mathcal{H}=(X, \mathcal{B}, \emptyset)$. In this case, we will use the notation $C S(t, k, v)$ or $C S T S(v)$ or $C S Q S(v)$.

- Finally, when all the blocks are bi-edges, we consider a bi-hypergraph of the type $\mathcal{H}=(X, \mathcal{B}, \mathcal{B})$. In this case, we will use the notation $B S(t, k, v)$ or $B S T S(v)$ or $B S Q S(v)$.

Evidently, all $S T S, S Q S$, and all $C S T S, C S Q S$ are colorable. The upper chromatic number of $C S T S(v)$ is fairly well understood; for instance, if $v \leq 2^{k}-1$, then $\bar{\chi}(C S T S(v)) \leq k$, and the cases of equality are structurally characterized (Milazzo, Tuza [35, 36]). More generally, $\bar{\chi}(C S(k-1, k, v))=$ $O(\ln v)$ holds for every fixed $k$; but if $t \leq k-2$, then $\bar{\chi}(C S(k-1, k, v))$ grows at least as fast as $v^{c}$ for some constant $c>0$ (Milazzo, Tuza [37]).

However, some $B S T S, B S Q S$ may be uncolorable. More specifically, there are uncolorable systems $B S T S$ for all orders at least 15 , see [19, 35]. This information and discussions with Alex Rosa (1998) gave rise to the idea 
that it may very well be that almost all triple systems $B S T S$ are uncolorable. On the other hand, no uncolorable $B S Q S$ has been found yet. For small orders it is known [33] that all $B S Q S$ on $v \leq 16$ vertices are colorable.

An interesting discovery was that some $P_{3}$-designs have gaps in their chromatic spectra, (L. Gionfriddo [20]). It raises questions about the existence of other designs with or without gaps in their chromatic spectra. M. Gionfriddo conjectured in [21] that every BSTS has a gap-free chromatic spectrum.

A survey on colorings of mixed Steiner systems can be found in [38]. Very recently, it has been proved by Bacsó and Tuza in [1] that the best possible general upper bound for the upper chromatic number of finite projective planes of order $q$ is equal to $q^{2}-q-\Theta(\sqrt{q})$ as $q$ tends to infinity, both when considered as $\mathcal{C}$ - and bi-hypergraphs.

\section{Open problems.}

1. Prove or disprove that almost all $B S T S$ are uncolorable.

2. Prove or disprove that all BSTS have gap-free chromatic spectrum.

3. Find the order of the smallest uncolorable $B S Q S$ (if there are any).

4. Do there exist $B S Q S$ with arbitrarily large upper chromatic number?

5. Do there exist $B S Q S$ with arbitrarily large lower chromatic number?

6. Determine $\lim \sup _{v \rightarrow \infty} \bar{\chi}(C S Q S(v)) / \log _{2} v$ taken over Steiner quadruple systems as $\mathcal{C}$-hypergraphs. Is this limit equal to 1 ?

7. For $k \geq t+2 \geq 4$, determine the largest possible exponent $c=c(k, t)$ such that $\bar{\chi}(S) \geq \Omega\left(v^{c}\right)$ for all Steiner systems $S=C S(t, k, v)$.

8. Estimate the upper chromatic number of lines and of higher subspaces in the finite projective and affine spaces viewed as $\mathcal{C}$ - or bi-hypergraphs, as a function of order and dimension.

\section{More General Structures}

In this concluding section we mention some recent concepts that are generalizations of, or closely related to mixed hypergraphs. We are going to proceed with those structure classes according to a decreasing generality. At the time of writing this survey, all related manuscripts are still in the 
process of publication. Only few selected results will be mentioned from them, just to illustrate the flavor of those studies.

To a wide extent, the most general model is that of pattern hypergraphs, introduced by Dvořák et al. [17]. Each edge is associated with the family of its feasible color partitions - without any a priori restrictions on those feasible families - and a coloring of the hypergraph is proper if its color classes induce a feasible partition on each edge. Despite that the model is extremely general, a necessary and sufficient condition can be given for the presence of gaps in the chromatic spectrum. The characterization is established by identifying four special classes of edge patterns.

In the more specified but still fairly general concept of stably bounded hypergraphs $[9,10]$ the patterns are restricted by four functions $\boldsymbol{s}, \boldsymbol{t}, \boldsymbol{a} \boldsymbol{b}$ on the edges, with the following meaning. A coloring is proper if, in each edge $E$, the largest cardinality of a monochromatic subset is at least $\boldsymbol{a}(E)$ and at most $\boldsymbol{b}(E)$, whereas the largest cardinality of a totally multicolored subset is at least $\boldsymbol{s}(E)$ and at most $\boldsymbol{t}(E)$. The subclass of color-bounded hypergraphs (introduced in [5] under a different name) assumes the functions $\boldsymbol{s}$ and $\boldsymbol{t}$ only, but it still includes the mixed hypergraphs by $s(E)=2$ (with the trivial bound $\boldsymbol{t}(E)=|E|$ ) for $\mathcal{D}$-edges, $\boldsymbol{t}(E)=|E|-1$ (with $\boldsymbol{s}(E)=1$ ) for $\mathcal{C}$-edges, and both $\boldsymbol{s}(E)=2$ and $\boldsymbol{t}(E)=|E|-1$ for bi-edges.

Concerning the various combinations of the four possible color-bound functions there have been investigations into their interrelations with respect to the possible gaps in feasible sets, the hierarchy of the sets of chromatic polynomials, and substantial differences in the time complexity of unique $(n-1)$-colorability $([7,9])$; moreover, the unexpectedly rich family of feasible sets for color-bounded hypertrees and efficient algorithms on various subclasses of stably bounded interval hypergraphs and hypertrees have been studied $([8,10])$.

Drgas-Burchardt and Łazuka [16] have investigated the chromatic polynomials for the class of hypergraphs that can be expressed, using the notation above, with the function $s$ alone. That is, the number of colors is lowerbounded in each edge, but no other types of restrictions are considered. In the paper [16], which was a substantial motivation also for introducing the generalizations above, the chromatic polynomial $P(\mathcal{H}, \lambda)$ has been investigated. It has been proved that there exists a strict connection between the coefficients of $P(\mathcal{H}, \lambda)$ and the sizes of the edges of $\mathcal{H}$. Consequences concerning the combinatorial meaning of the first three coefficients have been derived. (It is easy to see that the chromatic polynomial has order $n$, and the leading coefficient always equals 1 . These properties remain valid in 
the more general model involving the two color-bound functions $\boldsymbol{s}$ and $\boldsymbol{b}$ together, but fail already in the class of mixed hypergraphs.)

\section{REFERENCES}

[1] G. Bacsó and Zs. Tuza, Upper chromatic number of finite projective planes, J. Combin. Designs, to appear.

[2] G. Bacsó, Zs. Tuza and V. Voloshin, Unique colorings of bi-hypergraphs, Australasian J. Combin., 27 (2003), 33-45.

[3] C. Berge, Hypergraphs: Combinatorics of Finite Sets, North Holland (1989).

[4] Cs. Bujtás and Zs. Tuza, Orderings of uniquely colorable mixed hypergraphs, Discrete Applied Math., 155 (2007), 1395-1407.

[5] Cs. Bujtás and Zs. Tuza, Mixed colorings of hypergraphs, Electronic Notes in Discrete Mathematics, 24 (2006), 273-275.

[6] Cs. Bujtás and Zs. Tuza, Uniform mixed hypergraphs: The possible numbers of colors, Graphs Combin., to appear.

[7] Cs. Bujtás and Zs. Tuza, Color-bounded hypergraphs, I: General results. Manuscript (2006).

[8] Cs. Bujtás and Zs. Tuza, Color-bounded hypergraphs, II: Interval hypergraphs and hypertrees. Manuscript (2006).

[9] Cs. Bujtás and Zs. Tuza, Color-bounded hypergraphs, III: Model comparison, Applicable Analysis and Discrete Math., 1 (2007), 36-55.

[10] Cs. Bujtás and Zs. Tuza, Color-bounded hypergraphs, IV: Stable colorings of hypertrees (in preparation).

[11] Cs. Bujtás and Zs. Tuza, C-perfect hypergraphs (in preparation).

[12] E. Bulgaru and V. Voloshin, Mixed interval hypergraphs, Discrete Applied Math., 77(1) (1997), 24-41.

[13] M. Chudnovsky, N. Robertson, P. Seymour and R. Thomas, The strong perfect graph theorem, Annals of Math., 164(1) (2006), 51-229.

[14] K. Diao, P. Zhao and H. Zhou, About the upper chromatic number of a cohypergraph, Discrete Math., 220 (2000), 67-73.

[15] K. Diao, G. Liu, D. Rautenbach and P. Zhao, A note on the least number of edges of 3-uniform hypergraphs with upper chromatic number 2, Discrete Math., 306 (2006), 670-672.

[16] E. Drgas-Burchardt and E. Łazuka, On chromatic polynomials of hypergraphs, Applied Math. Letters, to appear.

[17] Dvořák, Z., J. Kára, D. Král' and O. Pangrác, Pattern hypergraphs. Manuscript (2004). 
[18] E. Flocos, Proprietati cromatice ale co-monostelelor, Buletinul Academiei de Stiinte a RM, Matematica, Chisinau (1997), No. 3, pp. 8-19. (in Romanian).

[19] B. Ganter, private communication (1997).

[20] L. Gionfriddo, Voloshin's colourings of $P_{3}$-designs, Discrete Math., 275 (2004), 137-149.

[21] M. Gionfriddo, Colourings of hypergraphs and mixed hypergraphs, Rendiconti del Seminario Matematico di Messina. Serie II, Tomo XXV, Vol. 9 (2003), 87-98.

[22] M. Gionfriddo, L. Milazzo and V. Voloshin, On the upper chromatic index of a multigraph, Computer Sci. J. Moldova, 10(1) [28] (2002), 81-91.

[23] T. Jiang, D. Mubayi, V. Voloshin, Zs. Tuza and D.B. West, The chromatic spectrum of mixed hypergraphs, Graphs Combin., 18 (2002), 309-318.

[24] D. Kobler and A. Kündgen, Gaps in the chromatic spectrum of face-constrained plane graphs, Electron. J. Combin., 8(1) (2001), \#N3.

[25] D. Král, A counter-example to Voloshin's hypergraph co-perfectness conjecture, Australasian J. Combin., 27 (2003), 25-41.

[26] D. Král, On feasible sets of mixed hypergraphs, Electron. J. Combin., 11(1) (2004), \#R19.

[27] D. Král', Mixed Hypergraphs and Other Coloring Problems, Discrete Math., 307(7-8) (2007), 923-938.

[28] D. Král, J. Kratochvíl, A. Proskurowski and H.-J. Voss, Coloring mixed hypertrees, Discrete Applied Mathematics, 154(4) (2006), 660-672.

[29] D. Král', J. Kratochvíl and H.-J. Voss, Mixed hypergraphs with bounded degree: edge-coloring of mixed multigraphs, Theoretical Computer Science, 295(1-3) (2003), 263-278.

[30] D. Král', J. Kratochvíl and H.-J. Voss, Mixed hypercacti, Discrete Math., 286(1-2) (2004), 99-113.

[31] J. Kratochvíl, Zs. Tuza and M. Voigt, New trends in the theory of graph colorings: Choosability and list coloring, in: Contemporary Trends in Discrete Mathematics (R. L. Graham et al., eds.), DIMACS Series in Discrete Mathematics and Theoretical Computer Science, 49, Amer. Math. Soc. (1999), pp. 183-197.

[32] A. Kündgen, E. Mendelsohn and V. I. Voloshin, Coloring of planar mixed hypergraphs, Electron. J. Combin., 7 (2000), \#R60.

[33] G. Lo Faro, L. Milazzo and A. Tripodi, The first BSTS with different lower and upper chromatic numbers, Australasian J. Combin., 22 (2000), 123-133.

[34] D. Lozovanu and V. Voloshin, Integer programming models for colorings of mixed hypergraphs, Computer Sci. J. Moldova, 8 (2000), 64-74.

[35] L. Milazzo and Zs. Tuza, Upper chromatic number of Steiner triple and quadruple systems, Discrete Math., 174 (1997), 247-259.

[36] L. Milazzo and Zs. Tuza, Strict colourings for classes of Steiner triple systems, Discrete Math., 182 (1998), 233-243. 
[37] L. Milazzo and Zs. Tuza, Logarithmic upper bound for the upper chromatic number of $\mathrm{S}(t, t+1, v)$ systems. Manuscript (2004).

[38] L. Milazzo, Zs. Tuza and V. I. Voloshin, Strict colorings of Steiner triple and quadruple systems: a survey, Discrete Math., 261 (2003), 399-411.

[39] A. Niculitsa and V. Voloshin, About uniquely colorable mixed hypertrees, Discuss. Math. Graph Theory, 20(1) (2000), 81-91.

[40] A. Niculitsa and H.-J. Voss, A characterization of uniquely colorable mixed hypergraphs of order $n$ with upper chromatic numbers $n-1$ and $n-2$, Australasian J. Combin., 21 (2000), 167-177.

[41] V. Prisakaru, The upper chromatic number of quasi-interval co-hypergraphs, Le Mathematiche, LII (1997) - Fasc. II, 237-260.

[42] Zs. Tuza, Graph colorings with local constraints - A survey, Discuss. Math. Graph Theory, 17(2) (1997), 161-228.

[43] Zs. Tuza and V. I. Voloshin, Uncolorable mixed hypergraphs, Discrete Applied Math., 99 (2000), 209-227.

[44] Zs. Tuza, V. I. Voloshin and H. Zhou, Uniquely colorable mixed hypergraphs, Discrete Math., 248 (2002), 221-236.

[45] V. I. Voloshin, The mixed hypergraphs, Computer Sci. J. Moldova, 1 (1993), 45-52.

[46] V. I. Voloshin, On the upper chromatic number of a hypergraph, Australasian J. Combin., 11 (1995), 25-45.

[47] V. I. Voloshin, Coloring Mixed Hypergraphs: Theory, Algorithms and Applications, Fields Institute Monograph, Amer. Math. Soc. (2002).

[48] V. Voloshin, Mixed Hypergraph Coloring Web Site: http://spectrum.troy.edu/ voloshin/mh.html

[49] V. Voloshin and H.-J. Voss, Circular mixed hypergraphs I: colorability and unique colorability, in: Proceedings of the Thirty-first Southeastern International Conference on Combinatorics, Graph Theory and Computing, Boca Raton, FL, 2000, Congr. Numer., 144 (2000), pp. 207-219.

[50] V. I. Voloshin and H. Zhou, Pseudo-chordal mixed hypergraphs, Discrete Math., 202 (1999), 239-248.

[51] A. A. Zykov, Hypergraphs, Uspekhi Mat. Nauk, 29 (1974), 89-154. (in Russian)

\section{Zsolt Tuza}

Computer and Automation Institute Hungarian Academy of Sciences

H-1111 Budapest, Kende u. 13-17

Hungary
Vitaly Voloshin

Department of Mathematics and

Physics

Troy University

Troy, AL 36082

U.S.A 